\title{
LOS ESTÁNDARES DE APRENDIZAJE DE LA LOMCE: ¿MEJORARÁN LA ENSEÑANZA Y EL APRENDIZAJE DE HISTORIA?
}

\author{
Carlos Fuster García ${ }^{1}$ \\ Facultat de Magisteri - Universitat de València
}

Recibido: 29/10/2014

Aceptado: 23/4/2015

\section{RESUMEN}

El potencial que pueden adoptar los estándares de aprendizaje esta relacionado con la forma y el uso que se haga de ellos, pudiendo mejorar la evaluación educativa, o por el contrario, creando modelos de aprendizaje donde se enseñe aquello que solo es evaluable, condicionando los conocimientos a aprender.

Este artículo analiza los estándares de aprendizaje enunciados en el borrador del currículum para la enseñanza de Historia de España de $2^{\circ}$ de Bachillerato. Como elemento de comparación, se describen los efectos que sobre el aprendizaje del alumnado provocan las pruebas de acceso a la Universidad (PAU), en nuestro caso, de la Universitat de València. Finalmente, se plantea una propuesta para la formulación de estándares de aprendizaje de Historia.

Palabras clave: conocimiento histórico - estándares de aprendizaje - evaluación - pruebas externas - currículo

\section{ABSTRACT}

The potential can adopt learning standards is related to the shape and the use made of them, they can improve the educational assessment, or conversely, create models of segregating evaluation. This paper examines the learning standards formulated in the draft curriculum for teaching History of Spain. As a point of comparison, this paper describes the effects on student learning cause the University Access Test, in our case, of the University of Valencia. Finally, it presents a proposal for the formulation of History standards of learning.

Keywords: historical knowledge - learning standards - assessment - external testing education program

\footnotetext{
${ }^{1}$ fuster.garcia.carlos@gmail.com

Estudiante del Programa de Doctorado en Didácticas Específicas, Universitat de València y miembro del Proyecto Gea-Clío. Este artículo deriva de una tesis doctoral en curso en Didáctica de las Ciencias Sociales dirigida por Xosé Manuel Souto.
} 


\section{INTRODUCCIÓN}

Es innegable que la cultura evaluativa se ha convertido en el elemento vertebrador del sistema educativo, llegándose a entender ésta como sinónimo de certificado de ciertos estándares educativos definidos previamente y medibles a través de evaluaciones externas del sistema. Un ejemplo de ello, es la incorporación de estándares de aprendizaje evaluables y evaluaciones externas de fin de etapa (reválidas) en la nueva ley de educación española (LOMCE).

Pocos dudan actualmente de que la práctica evaluativa acabe condicionando la acción en el aula, la selección de contenidos y las pruebas examinatorias elaboradas por el docente, así como la percepción social de la educación. Asimismo, a medida que nos introducimos en la educación obligatoria y postobligatoria, la subordinación se hace mayor, alcanzando un alto grado de intensidad con la celebración de las pruebas de acceso a la Universidad (PAU) por tratarse de pruebas externas, preparadas por personas que son miembros de la comunidad universitaria y distintas a los docentes que imparten las clases (Merchán, 2005) Paralelamente, las PAU adquieren una función de rendición de cuentas, que enfrenta al profesorado a un mecanismo que evalúa su práctica docente a partir del rendimiento de su centro.

Todo parece indicar que la PAU, tal y como la conocemos actualmente desaparecerá en el curso 2017-2018, sustituida ${ }^{2}$ por una reválida a modo de evaluación final de Bachillerato. En el caso que nos ocupa, será objeto de evaluación la asignatura de Historia de España en las tres modalidades que la ley contempla, y que a priori, para su elaboración, se tendrá en cuenta los criterios de evaluación y estándares de aprendizaje recogidos en el borrador del currículum de $2^{\circ}$ de Bachillerato.

Supuesto lo anterior ¿qué motivos han llevado al Ministerio de Educación para la introducción de estándares y reválidas? Según Ángel Pérez (2013) dos parecen ser los motivos: el elevado índice de fracaso y abandono escolar y el deterioro del nivel del sistema educativo como demostrarían los resultados de PISA.

\footnotetext{
${ }^{2}$ La calificación final de Bachillerato se obtendrá de la media de las calificaciones ponderada al 60\%, y de la evaluación final ponderada al 40\%, teniéndose en cuenta dicha nota para el acceso a la Universidad. Paralelamente, las Universidades podrán establecer procedimientos de admisión que quedan recogidos en la ley orgánica 8/2013, de 28 de febrero (LOMCE).
} 
Para explicar la introducción de los estándares de aprendizaje, la LOMCE señala "que los países que han mejorado de forma relativamente rápida la calidad de sus sistemas educativos han implantado medidas relacionadas con la simplificación del currículo”, todo parece indicar que estamos abocados a un currículo estándar y regulado hasta en sus mínimos detalles, no obstante, como afirma Darling-Hammond (2001) los currículums altamente prescriptivos no mejoran el aprendizaje del alumnado, y especialmente si se centran en el control de la enseñanza, un control que en el caso español estaría en relación con el incremento de pruebas y exámenes (Souto, Fita y Fuster, 2013). Por todo ello, no parece creíble que un aumento de la presión evaluativa vaya a reducir el fracaso o el abandono escolar. También podemos explicar cómo a través de los exámenes y reválidas se produce una segregación social, pues los más débiles abandonan el sistema escolar sin adquirir la categoría de ciudadanos (Fernández Enguita, 1987).

Por otra parte, intentar justificar la introducción de pruebas externas en comparación con el prestigio que han adquirido las pruebas PISA supone según Pérez (2013) "mala fe o ignorancia ciertamente culpable" puesto que como señala la OCDE (2005) la evaluación no mide el dominio de un programa escolar concreto, sino más bien la capacidad de utilizar los conocimientos y habilidades para hacer frente a problemas de la vida real, es decir, ir más allá de la mera transmisión de información. Es por ello que no abogamos por la eliminación de la evaluación, sino en el caso de la asignatura de Historia, creemos que es posible la existencia de pruebas externas que midan la adquisición de competencias históricas, dado que como plantea Domínguez (2013) es posible enriquecer este tipo de pruebas planteando competencias históricas específicas como se plantea en las científicas de PISA.

En resumen, en España se esta introduciendo una política educativa basada en la gestión empresarial de la escuela ${ }^{3}$ (Merchán, 2011, 2012), un intento de trasladar el modelo fabril de la producción en cadena al sistema educativo. Un modelo que entiende la educación como una inversión en capital humano, que tiene como objetivo principal

\footnotetext{
${ }^{3}$ En las aportaciones de Javier Merchán $(2011,2012)$ se analiza detalladamente los fundamentos en los que se apoyan estás políticas poniendo como ejemplo el caso de Andalucía.
} 
la mejora de la eficacia escolar y que necesita de indicadores medibles y evaluables para determinar la estrategia a definir.

Estas connotaciones alrededor de la evaluación, junto con los resultados obtenidos en un estudio de caso en torno a los resultados obtenidos por los estudiantes en las PAU de Historia de España de la Universitat de València (Fuster, 2013; Sáiz, 2013; Sáiz y Fuster, 2014) y ante la ausencia de evaluaciones externas que valoren la adquisición de competencias históricas, aconseja analizar y evaluar las posibles consecuencias que sobre la enseñanza y aprendizaje de Historia tendrá la implantación de la LOMCE.

\section{EL APRENDIZAJE DE HISTORIA A PARTIR DE LOS ESTÁNDARES DE APRENDIZAJE DE LA LOMCE}

La introducción de los estándares de aprendizaje viene avalada por la necesidad de mejorar la eficacia y la calidad de la educación escolar a partir de la evaluación del rendimiento del alumnado. No obstante, los resultados de las investigaciones no establecen un consenso a este respecto a nivel internacional (Barnes, Clark y Stephens, 2000; Darling-Hammond, 2001; Haymore Sandholtz y Paredes Scribner, 2004, Sheldon y Biddle, 1998; Solomon, 2003) y nacional (Coll y Martí, 2006; Merchán, 2005; Álvarez, 2003; Salinas, 2002) Si que creemos, por otra parte, que el planteamiento de unas pruebas que no midan únicamente el éxito sino que sean relevantes para que los alumnos demuestren la adquisición de destrezas y competencias puede resultar útil (Fuster, 2013) al mismo tiempo que pueden llevar adscritos procesos de innovación educativa (Souto 2011; Souto y Claudino, 2001) sobre todo teniendo en cuenta la cautela con la que debemos enfrentarnos a los resultados obtenidos a partir de las pruebas externas estandarizadas (Popham, 1999; Sanmartí, 2003).

A pesar de la falta de acuerdo, y aunque los estándares y la evaluación no pueden por si mismos guiar la práctica docente, si están bien diseñados y concitan un amplio consenso, pueden llegar a potenciar el aprendizaje de los estudiantes; no obstante, dependerá del uso y de la forma que adopten (Darling-Hammond, 2001). Un 
reciente artículo publicado por Ramón López Facal ${ }^{4}$ (2014) nos obliga a ser cautos en la influencia que ejercerán los estándares sobre el aprendizaje de Historia.

En el caso español ¿cómo se definen los estándares de aprendizaje? ¿qué forma adoptan los estándares de la materia de Historia de España de $2^{\circ}$ de Bachillerato?

El Ministerio de Educación define los estándares de aprendizaje evaluables como:

Especificaciones de los criterios de evaluación que permiten definir los resultados de aprendizaje, y que concretan lo que el alumno debe saber, comprender y saber hacer en cada asignatura: deben ser observables, medibles y evaluables y permitir graduar el rendimiento o logro alcanzado. Su diseño debe contribuir y facilitar el diseño de las pruebas estandarizadas y comparables.(Real Decreto 126/2014) ${ }^{5}$

El potencial de los estándares como instrumentos de orientación son diversos, pero hay tres cuyo papel parece determinante. El primero es el de determinar los contenidos, es decir, definir qué es lo que los alumnos deberían saber. Dichos estándares nos definen que historia se debe enseñar y aprender, y por tanto, de manera implícita o no, nos transmite el modelo escolar relacionado con el aprendizaje de la Historia. El conocimiento histórico que definan los estándares pueden contribuir a desarrollar un aprendizaje crítico que permita al alumnado a poner en marcha las competencias históricas que ayuden a integrar el conocimiento y orientarlo hacia la comprensión de problemas del pasado y del presente, o por el contrario, pueden orientar el aprendizaje hacia una actividad mecánica de repetición y memorización, que acabe convirtiendo el conocimiento histórico es un discurso unívoco e inservible. El segundo, los criterios de rendimiento, a saber, cómo deberían demostrar que han adquirido el conocimiento. Sirven para precisar los criterios de evaluación y proporcionar ejemplos e

\footnotetext{
${ }^{4}$ En un reciente artículo, Ramón López Facal (2014) analiza los contenidos de historia recogidos en el proyecto oficial publicado tras la aprobación de la LOMCE, concluyendo que se trata de un modelo basado fundamentalmente en la memorización y donde la enseñanza no va a favorecer la capacidad de interpretación de los procesos históricos.

${ }^{5}$ Se ha consultado el Real Decreto 126/2014, de 28 de febrero, por el que se establece el currículo básico de la Educación Primaria por tratarse del único documento oficial donde se recoge la definición de estándar de aprendizaje. (BOE-A-2014-2222, pp. 19351-19352)
} 
indicadores, y a priori, estos deberían estar regulados por las competencias básicas. En otros términos, estos debería evaluar no tanto la mera transmisión del conocimiento, como por la capacidad de utilizarlo. Por último, la función de rendición de cuentas a partir de pruebas externas y que nos permita evaluar el grado de adquisición de dichos estándares. A este respecto, ya hemos tenido oportunidad de valorar los efectos que sobre la practica docente tiene la imposición de una prueba al final de un periodo educativo concreto, como puede ser el caso de la PAU. (Fuster, 2013a, 2013b; Souto, Fuster y Sáiz, 2014; Fita, Fuster y Souto, 2014)

Habiendo definido la singularidad de los estándares de aprendizaje, es preciso analizar cuál es la propuesta que se ha elaborado para la materia de Historia de España de $2^{\circ}$ de Bachillerato ${ }^{6}$. En ella, los contenidos abarcan desde el Paleolítico a la actualidad, organizados en 12 bloques y concretados en 174 estándares de aprendizaje.

Considerando la complejidad cognitiva que plantean los estándares de aprendizaje y siguiendo la taxonomía de objetivos-etapas de aprendizaje ${ }^{7}$ diseñada por Bloom, en su versión reciente y adaptada por Anderson y Krathwohl (2001) obtenemos los resultados observables en la Figura 1.

Como se desprende de dicha figura, y teniendo en cuenta dos de los factores definitorios del estándar de aprendizaje (contenido y rendimiento) observamos como 3 de cada 4 estándares (77’1\%) hace referencia al contenido y solo el 22’8\% al rendimiento. Si comparamos esta situación con una asignatura troncal de carácter instrumental, como Lengua Castellana y Literatura ${ }^{8}$, observamos una mayor presencia de estándares de carácter procedimental y metacognitivo. Se esta reduciendo, por tanto

\footnotetext{
${ }^{6}$ Para la redacción del artículo se ha consultado el Real Decreto 1105/2014, de 26 de diciembre, por el que se establece el currículo básico de la Educación Secundaria Obligatoria y del Bachillerato. BOE núm.3, de 3 de enero de 2015, páginas 169 a 546. BOE-A-2015-37.

${ }^{7}$ La practicidad de la taxonomía de Bloom, revisada por Anderson y Krathwohl, reside en que resulta fácilmente entendible para comprender el nivel cognitivo de los estándares. Así mismo, la taxonomía es utilizada como un elemento de apoyo y orientación para diferenciar la complejidad entre los mismos.

${ }^{8}$ De base la asignatura de Lengua Castellana y Literatura esta organizada en torno a las competencias lingǘísticas que se pretenden desarrollar: "Bloque 1. Comunicación oral: hablar y escuchar. Bloque 2. Comunicación escrita: leer y escribir. Bloque 3. Conocimiento de la Lengua. Bloque 4. Educación Literaria.”
} 
a la marginalidad asignaturas que no son consideradas fácilmente evaluables con estándares universales (López Facal, 2014) a pesar de las propuestas ya señaladas.

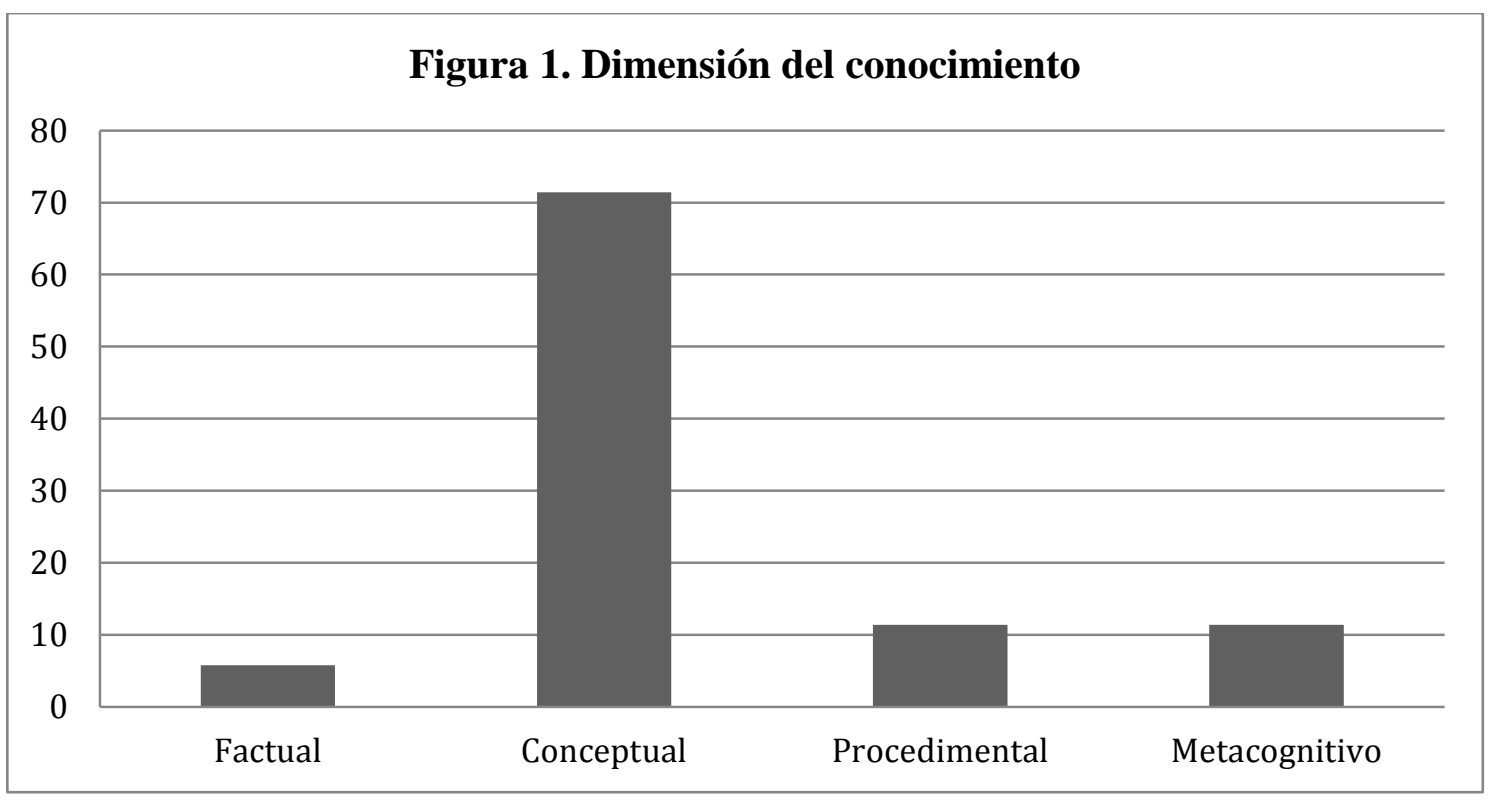

Fuente: elaboración propia a partir del análisis de los estándares de aprendizaje.

Respecto al conocimiento factual, resulta paradójico encontrarse en 9 de los bloques, estándares relacionados con la definición de conceptos ${ }^{9}$, de este modo, cada periodo histórico queda relacionado con un vocabulario específico. Más allá de cuestionar los términos a definir y su adecuación, nos preocupa la transmisión de una idea de la Historia de carácter “enciclopédico” y que lleva asociado procesos cognitivos basados en la memorización y el recuerdo.

En esta misma línea se sitúan los estándares de carácter conceptual, que debido a los contenidos extremadamente amplios, provoca que estos se traduzcan en tareas difícilmente abarcables por los alumnos y acaba convirtiéndose en el resumen o descripción de los hechos históricos más relevantes ${ }^{10}$. Se trata de estándares que

\footnotetext{
${ }^{9}$ En el bloque 2, "La Edad Media: tres culturas y un mapa político en constante cambio": "Define con precisión los siguientes términos o conceptos: califato, reinos de taifas, mozárabe, señorío, estamentos, Cortes.”

${ }^{10}$ En el bloque 3, "La formación de la monarquía hispánica y su expansión mundial” como ejemplo: Compara los imperios territoriales de Carlos I y el de Felipe II, y explica los diferentes problemas que acarrearon. Explica la expansión colonial en América y el Pacífico durante el siglo XVI. [...] Compara y comenta las rebeliones de Cataluña y Portugal.”
} 
accionan procesos cognitivos como la descripción, la explicación o el resumen, y que por tanto conlleva una única comprensión de los contenidos. A pesar de ello, continuamos estando situados en una dimensión baja de complejidad cognitiva.

En cuanto a los conocimientos de carácter procedimental, todos los bloques conllevan la elaboración de al menos un esquema, una búsqueda de información y una breve exposición de la misma, la representación de una línea del tiempo y el comentario de una fuente (textual, gráfica o estadística). Por tanto cada uno de ellos se realiza al menos una docena de veces a lo largo de todo el curso, lo que supone un exceso de actividades, y en ocasiones, de una complejidad y de inversión de tiempo que no garantizan la adquisición de conocimientos históricos rigurosos ni útiles (López Facal, 2014).

Por último, se ha señalado un 11’4\% de estándares de carácter metacognitivo. Cabe matizar que este porcentaje es matizable, dado que se ha tenido en cuenta que los estándares de carácter procedimental deberían llevar asociados prácticas como estrategias para la búsqueda de información, métodos de planificación de trabajo, estrategias para la comprensión de lectura... Del mismo modo que se ha valorado el carácter de los estándares respecto al tipo de conocimiento, es necesario hacerlo en relación a los procesos cognitivos activados. Entendemos que dichos estándares suponen, en muchas ocasiones, la necesidad de aplicar o analizar el conocimiento, entre otros, al realizar un comentario o al realizar un gráfico o esquema, así mismo, algunos de los estándares referidos suponen altos niveles de complejidad como la planificación y producción de narrativas. No obstante, creemos que la gran demanda de trabajo junto con la limitación temporal, provocará una falta de profundidad y rigor necesarios para abordar dichas cuestiones.

La propuesta realizada para la Historia de España de $2^{\circ}$ Bachillerato incide en presentar una historia general que excede los contenidos abarcables en un curso escolar. Los estándares de aprendizaje promueven un aprendizaje superficial donde no se profundiza en la adquisición de metaconceptos históricos. Si los estándares de aprendizaje van a formar parte de la evaluación externa que contempla la LOMCE, nos encontraremos con una prueba que no va a favorecer el desarrollo de las competencias históricas. Así mismo, si el modelo a seguir son las pruebas PISA, no consideramos que 
con los estándares propuestos vayan a favorecer la aplicación de conocimientos que es lo que realmente miden dichas pruebas. En esta misma línea, echamos en falta una mejor definición de las competencias que la Historia debería desarrollar ${ }^{11}$, así como una mayor descripción de los indicadores de logro de dichas competencias.

\section{ESTUDIO DE CASO: LAS PAU DE HISTORIA DE ESPAÑA DE LA COMUNITAT VALENCIANA.}

En España, con anterioridad a la instauración de las pruebas de diagnóstico, las pruebas externas por antonomasia, se vienen realizando desde hace más de treinta años, han sido las pruebas de acceso a la Universidad (PAU). Así ha dejado constancia el Consejo Escolar del Estado (2009) o las investigaciones impulsadas por el Centro de investigación y Documentación Educativa (CIDE).

Estas pruebas se convirtieron en la única evaluación externa del sistema, que permite contrastar las calificaciones obtenidas por los alumnos y la consecución de los objetivos de aprendizaje planteados. En este sentido, desempeñan un papel fundamental en el equilibrio de las enseñanzas compartidas y en la homologación de los distintos centros que las imparten, sean éstos públicos o privados. (Muñoz-Repiso et alíí., 1997:16)

Por ello hemos afirmado, y demostrado, en más de una ocasión que las PAU son una oportunidad para realizar un diagnóstico del aprendizaje realizado por el alumnado, y constituyen un referente para valorar la idoneidad del currículum (Coll y Martí, 2006). Además nos permite suponer las mejoras en la enseñanza para lograr una autonomía intelectual crítica y una madurez personal (Souto, 2011).

En la actualidad desconocemos como se van a plantear las pruebas externas de final de $2^{\circ}$ de Bachillerato propuestas en la LOMCE; no obstante, el diagnóstico realizado en torno a las PAU puede, al menos, anticipar los problemas que acarrearía un tipo de prueba que siguiera los estándares de aprendizaje propuestos en el borrador. Partimos de la hipótesis de que un tipo de prueba como la planteada en Junio de 2012 en

\footnotetext{
${ }^{11}$ A pesar de que los currículos se organizan por competencias no ocurre así en todos los niveles, como ocurre en el caso del Bachillerato (González, 2012)
} 
la Comunidad Valenciana podría resultar factible en el marco de los estándares analizados, como demostramos en el Cuadro 1.

Cuadro 1. Comparación de la PAU de Historia de España (Junio de 2012) y los estándares de aprendizaje propuestos.

\begin{tabular}{|c|c|}
\hline $\begin{array}{c}\text { Preguntas PAU de Historia de España } \\
\text { (Junio de 2012) }\end{array}$ & $\begin{array}{c}\text { Estándares de aprendizaje propuestos para } \\
\text { el bloque } 12 \text { (Normalización democrática } \\
\text { de España e integración en Europa) }\end{array}$ \\
\hline $\begin{array}{l}\text { 1. Describe el tipo de fuentes utilizadas en } \\
\text { los textos. }\end{array}$ & $\begin{array}{l}\text { "1.2. Describe el papel desempeñado por } \\
\text { el rey durante la transición.” }\end{array}$ \\
\hline $\begin{array}{l}\text { 2. Identifica el núcleo de ambos textos y } \\
\text { señala las ideas principales que se } \\
\text { desprenden de cada uno de ellos } \\
\text { situándolas en el núcleo correspondiente. }\end{array}$ & $\begin{array}{l}\text { "3.1. Elabora un esquema con las etapas } \\
\text { políticas desde } 1979 \text { hasta la actualidad, } \\
\text { según el partido en el poder, y señala los } \\
\text { principales" }\end{array}$ \\
\hline $\begin{array}{l}\text { 3. Explica los conceptos "golpe de } \\
\text { Estado" y "referéndum". } \\
\text { 4. Explica la dinámica política durante la } \\
\text { Transición democrática, explica el papel } \\
\text { que ha jugado la monarquía a lo largo del } \\
\text { siglo XX, desde su participación durante } \\
\text { las dictaduras y su actitud ante la } \\
\text { instauración de regímenes democráticos. }\end{array}$ & $\begin{array}{l}\text { “6.1. Representa una línea del tiempo } \\
\text { desde } 1975 \text { hasta 2011, situando en ellos } \\
\text { los principales acontecimientos } \\
\text { históricos” } \\
\text { “7.1. Comenta un texto, mapa, gráfico, } \\
\text { tabla o imagen o responde a cuestiones } \\
\text { planteadas a partir de ellos” }\end{array}$ \\
\hline
\end{tabular}

Fuente: elaboración propia.

Como podemos comprobar, la prueba planteada en Junio de 2012 se adapta a los estándares de aprendizaje propuestos para el bloque relacionado con la España democrática, es decir, la prueba se podría mantener inalterable si se acaba aprobando el borrador propuesto. 
No es objeto de este artículo el análisis exhaustivo del protocolo de examen ${ }^{12}$, nos centramos por tanto en los resultados observables en las respuestas del alumnado. Para facilitar la lectura de los mismos y comprobar la complejidad y coherencia del discurso de los alumnos se ha optado por utilizar la taxonomía SOLO desarrollada por Biggs y Tang (2007). En la taxonomía SOLO las respuestas de los alumnos forman una estructura de complejidad desde el simple reconocimiento pre y uniestructural a la relacional o abstracto ampliado. Se trata de niveles de complejidad creciente.

El cuadro 2 permite identificar las limitaciones detectadas en las respuestas del alumnado: la mayoría solo son capaces de reproducir memorísticamente los contenidos, mostrando un nivel de aprendizaje multiestructural (nivel 2), es decir, describen numerosos datos pero sin llegar a estructurarlos y sin establecer relaciones causales. En definitiva, la mayoría de respuestas se enmarcan entre el nivel uniestructural y relacional. De esto modo, 4 de cada 5 respuestas (78’8\%) se pueden tipificar entre los niveles 1,2 y 3.

Cuadro 2. Niveles de complejidad de respuesta de la PAU de Historia de la Comunitat Valenciana.

\begin{tabular}{|l|c|c|c|c|c|}
\hline Ejercicios & Nivel 0 & Nivel 1 & Nivel 2 & Nivel 3 & Nivel 4 \\
\hline Primero & 2 & 29 & 28 & 26 & 15 \\
\hline Segundo & 7 & 25 & 30 & 26 & 12 \\
\hline Tercero & 3 & 25 & 14 & 31 & 27 \\
\hline Cuarto (a) & 11 & 17 & 30 & 25 & 17 \\
\hline Cuarto (b) & 7 & 29 & 44 & 15 & 5 \\
\hline Total & $30(6 \%)$ & $125(25 \%)$ & $146(29,2 \%)$ & $123(24,6 \%)$ & $76(15,2)$ \\
\hline
\end{tabular}

Nivel 0: Preestructural. Nivel 1: Uniestructural. Nivel 2: Multiestructural. Nivel 3: Relacional. Nivel 4: Abstracto Ampliado

Fuente: elaboración propia.

A modo de ejemplo, nos detendremos a analizar algunas de las respuestas emitidas por el alumnado en la tercera y cuarta pregunta:

\footnotetext{
${ }^{12}$ Un análisis exhaustivo se ha realizado en el marco de una investigación en Didáctica de las Ciencias Sociales (Fuster, 2013) dirigida por Xosé Manuel Souto.
} 
En la tercera pregunta ${ }^{13}$ se pide al estudiante que realice la explicación de dos conceptos relacionales con el contexto histórico. Los criterios de corrección proponen una única respuesta ${ }^{14}$ a partir de la cual el alumnado obtendría la máxima puntuación, por el contrario, las respuestas de los alumnos reflejan niveles de aprendizaje distintos. Desde un nivel elemental:

Golpe de Estado: acción normalmente llevada a cabo por el ejército, en el cual se lleva a término la abolición de la constitución, además de las organizaciones constitucionales (Examen $n^{\circ}$ 850745)

a respuestas más complejas, que implican un conocimiento histórico adecuado para un nivel preuniversitario:

Golpe de Estado: alzamiento de un grupo con ideas comunes y contrarias al régimen que viven. Normalmente hay siempre miembros militares que forman la parte armada del golpe y libran las batallas en caso de haber y administran la represión si el golpe triunfa. En España se han vivido diversos intentos de golpe de Estado. El mencionado en los textos el 23 de febrero de 1981 a cargo de Tejero; otro conocido como "sanjuanada” en tiempo de la II República, en 1932 concretamente. Aunque no fue un golpe de Estado, es necesario comentar el intento de arrebatar el poder a Miguel Primo de rivera por parte de los partidos políticos dinásticos y fuerzas republicanas (Examen $n^{\circ} 850686$ )

La cuarta pregunta es, en teoría, la menos determinada por los criterios de corrección ${ }^{15}$, en ella los alumnos deberían ser capaces de construir una narrativa histórica personal a partir de los documentos y sus conocimientos históricos, dando muestra de un control del tiempo histórico al establecer relaciones temporales pasado-

\footnotetext{
${ }^{13}$ Se hace referencia a la segunda opción del examen donde se les señala a los alumnos que expliquen los conceptos “golpe de estado" y “referéndum”.

14 “El primero <<golpe de Estado>> se referirá al intento de socavar los fundamentos del orden vigente en el que la participación de la democracia recién instaurada.”

15 "En esta última pregunta la respuesta es muy abierta y permite diversos razonamientos correctos. Con ello se valorará si el alumnado sabe refundir datos e ideas procedentes del proceso de aprendizaje de la materia y los que proceden del análisis de las fuentes facilitadas.”
} 
presente-futuro y finalmente ser capaces de dar muestra de su pensamiento crítico. $\mathrm{O}$ por el contrario, podríamos encontrarnos con discursos elaborados previamente y por tanto con narrativas academicistas estandarizadas, donde no hay duda de veracidad y donde no se suscitan interrogantes.

A partir de los resultados recogidos en el Cuadro 2, solo una minoría alcanza un nivel de respuesta elevado, $17 \%$ y $5 \%$ respectivamente, en comparación con las respuestas clasificadas como multiestructurales.

Así, encontramos como los alumnos acaban simplificando el hecho histórico a describir:

“El papel de la Monarquía a lo largo del S.XX ha sido muy importante, primero A.XIII [sic] durante la dictadura de Primero de Rivera ya que con la caída del dictador también cayó la monarquía. Durante la II República no había monarquía, pero durante la dictadura de Franco con la Ley de Sucesión imponía a Juan Carlos I como Monarca, que fue educado por Franco” (Examen $\left.n^{\circ} 850745\right)$

El nivel 4 corresponde a respuestas de alumnos que son capaces de manejar el tiempo histórico como elemento estructurante y presentan una narrativa donde los hechos históricos tienen una relación causal. Por ejemplo:

“Alfonso XIII gobernó desde 1902 hasta 1931, era militarista y eso se vería reflejado en el futuro, porque cuando tuvo problemas optó por un gobierno militar. Tras ser implicado en la matanza del desembarco de Alhucemas, pidió a Primo de Rivera que hiciera un Golpe de Estado, al cual dio soporte [...]

Finalmente la monarquía, durante la dictadura de Franco se mantuvo al margen, pero colaboró con los reformistas para que el país transitara a la democracia” (Examen n850681)

A partir de los resultados obtenidos podemos afirmar que el alumnado acaba repitiendo, pero con errores, los discursos elaborados por los historiadores o profesorado, y acaban desarrollando narrativas donde se establece una relación de hechos aislados, por lo que no se acaba produciendo un aprendizaje comprensivo. La mayoría de estudiantes muestran un conocimiento estructurado en epígrafes, reducido a 
frases afirmativas sin ninguna explicación. En esta misma línea concluyen García, Jiménez y Moreno (2011) al analizar las PAU de Historia en Andalucía.

En general, los criterios de corrección promueven e imponen un tipo de respuesta que no fomenta el análisis, la argumentación ${ }^{16}$ y/o la crítica. A partir del análisis realizado (Fuster, 2013b) las preguntas inicialmente abiertas son funcionalmente cerradas al limitar las orientaciones del corrector. Salvando las distancias, muestran una tendencia similar a la señalada al analizar los estándares de aprendizaje.

\section{HACIA UNA DEFINICIÓN DE ESTÁNDARES DE APRENDIZAJE DE HISTORIA}

La Didáctica de las Ciencias Sociales parece haber superado los rutinas escolares instauradas en relación al aprendizaje de historia. En la actualidad, el aprendizaje memorístico ha sido cuestionado y se han aportado alternativas al respecto desde campos y países diferentes ${ }^{17}$. Estos planteamientos coinciden en señalar la necesidad de una enseñanza-aprendizaje de historia que transcienda un aprendizaje por acumulación de información, hechos, fechas o personajes e integrar el conocimiento y uso de destrezas para acceder, comprender e interpretar ese pasado. No se trata de vaciar de contenido el aprendizaje histórico a favor de un aprendizaje competencial, sino combinar el aprendizaje de contenidos históricos (conceptos de primer orden) con el de competencias históricas (conceptos de segundo orden o metaconceptos).

Este replanteamiento (rethinking) de la enseñanza de historia ha sido formulado desde EEUU y Canadá en los últimos quince años en el contexto del Centre for the

\footnotetext{
${ }^{16}$ Una reciente investigación elaborada por Carmen Machí (2014) en la Universitat de Valencia, analiza las insuficiencias que los libros de texto de Ciencias Sociales tienen respecto al tratamiento de la argumentación, además identifica las dificultades que el alumnado tiene para elaborar argumentos razonados sobre el pasado reciente y su relación con el presente.

${ }^{17}$ Se aportan las referencias más significativas: Barca, 2000; Carretero y Voss, 2004; González et al. 2011; Gómez et al. 2014; Lee, 2005; Lévesque, 2008; López Facal et al. 2011; Pagès, 2009; Plà, 2005 y 2012; Prats y Santacana, 2011; Santisteban, 2010; Rüsen, 2005; Sáiz, 2013; Santiesteban et al. 2010; Seixas y Morton, 2013; Schmidt y Barca, 2009; Taylor y Young, 2003; Tribó, 2005; VanSledright, 2011 y 2014; Wineburg, 2001.
} 
Study of Historical Consciousnes. Definiéndose lo que se conoce como pensamiento histórico, un proceso creativo que realizan los historiadores para interpretar las fuentes del pasado y generar las narrativas históricas (Seixas y Morton, 2012). Así el pensamiento histórico se construye alrededor de seis conceptos que pueden ayudar a estudiantes y docentes a pensar como los historiadores transforman el pasado y construyen historia ellos mismos. Dichos conceptos no funcionan independientemente, sino que trabajan conjuntamente como partes del proceso de pensamiento y suponen decidir qué es importante estudiar del pasado (relevancia histórica), aprender a seleccionar, contextualizar e interpretar los hechos históricos a partir de fuentes históricas (pruebas históricas), entender la complejidad histórica y el impacto de los hechos históricos (cambio-continuidad, causa-consecuencia) aprehender el pasado desde la óptica de su contexto histórico (perspectiva histórica) y por último, desarrollar una conciencia crítica (dimensión ética).

Por tanto, ¿es posible plantear un currículum de Historia basado en las premisas del pensamiento histórico? El planteamiento e introducción de unos estándares de aprendizaje que promuevan no solo la adquisición de conocimiento conceptual, sino que también faciliten el desarrollo de competencias históricas puede favorecer un cambio curricular.

En el Cuadro 3 se plantea una propuesta inicial ${ }^{18}$ de la estructura a partir de la cual se podría desarrollar el currículum de Historia. Se plantean cuatro grandes bloques similares al modelo presentado en las asignaturas instrumentales. En el primer bloque, se abordarían los contenidos históricos (conceptos de primer orden) se trata de conocer y comprender los conceptos históricos (hechos, fechas, procesos, ideas...) relacionados con el tema abordado. En el segundo bloque, se valora la adquisición de las habilidades de pensamiento histórico anteriormente citadas. Su adquisición y progresión por parte de los estudiantes ha sido abordado por diversos autores ${ }^{19}$ y nos puede resultar de ayuda para evaluar su aprendizaje. En el tercer bloque, se trabaja la expresión y organización

\footnotetext{
${ }^{18}$ Se presenta una propuesta en fase de construcción, su forma definitiva será presentada cuando culmine el proceso de investigación doctoral.

${ }^{19}$ Respecto a la relevancia histórica contamos con las investigaciones de Cercadillo (2001) quien aborda como los estudiantes progresan desde un análisis presentista de la historia hacia análisis más elaborados de conciencia histórica. Por su parte, Sáiz (2013) ha elaborado una propuesta de marcadores en el uso de fuentes históricas textuales que nos permiten determinar el uso que hace el alumnado respecto de las mismas.
} 
de las ideas a través de narrativas históricas. El alumnado debe ser capaz de elaborar sus propios discursos narrativos en diferentes formatos (oral o escritos) y a diferentes audiencias. Por último, el cuarto bloque, la capacidad para transferir no solo el conocimiento sino también las competencias en diferentes contextos, en resumen, que se produzca un verdadero aprendizaje significativo.

Cuadro 3. Propuesta de currículum de Historia.

\begin{tabular}{|c|}
\hline BLOQUE 1. CONOCIMIENTOS HISTÓRICOS. \\
Adquisición del contenido histórico y comprensión de su significado \\
\hline BLOQUE 2. PENSAMIENTO HISTORICO. \\
Uso de habilidades de pensamiento histórico. \\
BLOQUE 3. COMUNICACIÓN. \\
Expresión y organización de las ideas e información (narrativas históricas) \\
\hline BLOQUE 4. APLICACIÓN. \\
Uso del conocimiento histórico y las competencias históricas para hacer conexiones \\
entre contextos diversos.
\end{tabular}

Fuente: elaboración propia a partir de la adaptación del currículum de la provincia de Ontario (Canadá).

Los cuatro bloques formulados deben ser considerados interrelacionados, reflejando la necesaria interconexión del aprendizaje. Los bloques ayudan a alumnos y docentes no sólo a plantear la adquisición del conocimiento sino también el desarrollo de las habilidades de pensamiento histórico, elaboración de narrativas históricas y aplicación del aprendizaje. Un posterior desarrollo debería determinar los grados de adquisición de cada uno de los bloques, permitiendo evaluar su grado de consecución por parte del alumnado.

\section{CONCLUSIONES}

La formulación de los estándares de aprendizaje de Historia de $2^{\circ}$ de Bachillerato parecen indicar un retroceso respecto a la introducción de las competencias en el currículum ya que suponen la adquisición de componentes conceptuales como única forma de aprendizaje, perjudicando el desarrollo de un aprendizaje crítico, y no permitiendo la puesta en marcha de las competencias históricas que proporcionen al 
alumnado las herramientas necesarias para comprender los problemas sociales del pasado y el presente.

Así mismo, si asumimos los resultados obtenidos de la investigación de la PAU de Historia de España (Fuster, 2013b; Sáiz, 2013 y Sáiz y Fuster, 2014) a modo de conjetura en relación a las futuras evaluaciones externas, todo parece indicar una deficiente alfabetización histórica. Tanto la propuesta de estándares de aprendizaje como el examen propuesto por las universidades valencianas dejan poco margen para acabar con el abuso de la memorización. En ambos casos se obvia el rendimiento de los alumnos, es decir, los diferentes niveles de complejidad que los alumnos pueden alcanzar, lo que acaba resumiéndose en una estandarización del conocimiento.

Debemos superar las rutinas escolares que han entendido el aprendizaje de historia como la simple memorización de contenidos e informaciones históricas de narrativas académicas. Las Ciencias Sociales deben apostar por el desarrollo de competencias que permitan la formación de una ciudadanía capacitada para abordar los problemas sociales y ambientales con los que se enfrentará en el futuro.

\section{BIBLIOGRAFÍA}

ÁLVAREZ, J.M. (2003). La evaluación a examen. Madrid: Miño y Dávila.

ANDERSON, L.W. y KRATHWOHL, D.R. (eds.) (2001). A taxonomy for Learning, teaching, and assessing: A revision of Bloom's taxonomy of educational objectives. Nueva York: Longman.

BARCA, I. (2000). O pensamento histórico dos jovens. Braga: Universidade do Minho.

BARNES, M.; CLARK, D. y STEPHENS, M. (2000). Assessment: the engine of systemic curricular reform? Journal of Curriculum Studies, 32, 5, 623-650.

BIGGS, J. y TANG. C. (2007), Teaching for Quality Learning at University. What the Students Does. Berkshire: McGraw-Hill.

CARRETERO, M. y VOSS, J. (comp.) (2004). Aprender y pensar la historia. Buenos Aires: Amorrortu.

CERCADILLO, L. (2001). Significance in history: student's ideas in England and Spain. En A. Dickinson, P. Gordon, y P. Lee (Eds.), Raising Standards in 
History Education. Internacional Review of History Education, Vol. 3 (pp. 116-145). London: Woburn Press.

COLL, C. y MARTÍN, E. (2006) Vigencia del debate curricular. Aprendizajes básicos, competencias y estándares. II Reunión del Comité Intergubernamental del Proyecto Regional de Educación para América Latina y el Caribe (PRELAC). Santiago de Chile. 11-13 de mayo de 2006. Documento no publicado. http://www.ub.edu/grintie

CONSEJO ESCOLAR DEL ESTADO (2009). Informe sobre el estado y situación del sistema educativo. Curso 2007-2008. Madrid: Ministerio de Educación.

DARLING-HAMMOND, L. (2001). El derecho de aprender. Crear buenas escuelas para todos. Barcelona: Ariel.

DOMÍNGUEZ, J. (2013). ¿Es posible evaluar competencias históricas como lo haría PISA? Íber. Didáctica de las Ciencias Sociales, Geografía e Historia, 74, 6274.

FERNANDEZ ENGUITA, M. (1987) Sistema de enseñanza y clases sociales. En C. Lerena (ed.), Educación y sociología en España. (pp. 195-306). Torrejón de Ardoz: Akal

FUSTER, C. (2013a). Las pruebas de acceso a la Universidad y el control del aprendizaje de Historia. En J. Prats, I. Barca, y R. López Facal (Eds.), Historia e Identidades culturales. Vol. 1 (pp. 1010-1021). Barcelona: AUPDCS.

FUSTER, C. (2013b). ¿Control o desarrollo del pensamiento histórico? Las PAU de Historia de España. Trabajo Fin de Máster de investigación en Didáctica de las Ciencias Sociales, inédito. Valencia: Universitat de València

GARCÍA, C.R.; JIMÉNEZ, M.D. y MORENO, C. (2011). Las pruebas de acceso a la universidad (PAU) de Historia de España. Una indagación sobre la formación del pensamiento histórico de los estudiantes. En P. Miralles, S. Molina y A. Santisteban (eds.) La evaluación en el proceso de enseñanza y aprendizaje de las ciencias sociales, Vol.1. (pp. 53-72) Murcia: APUDCS

GÓMEZ, C., ORTUÑO, J. y MOLINA, S. (2014), Aprender a pensar históricamente. Retos para la historia en el siglo XXI, Tempo e Argumento, 6 (11), 5-27.

GONZÁLEZ, I. (2012) Un Bachillerato sin competencias. Íber. Didáctica de las 
ciencias Sociales, Geografía e Historia, 72, 71-78

GONZÁLEZ, N., PAGÈS, J. y SANTISTEBAN, A. (2011). ¿Cómo evaluar el pensamiento histórico del alumnado? En P. Miralles, S. Molina, A. Santisteban (eds.), La evaluación y el proceso de enseñanza y aprendizaje de las ciencias sociales, vol. I, (pp. 221-232). Murcia: AUPDCS.

HAYMORE SANDHOLTZ, J.; OGAWA, R.T. y PAREDES SCRIBNER, S (2004). Standars gaps: unintended consequences of local standard-based reform. Teachers College Record, 106, 6, 1177-1202.

LEE, P. (2005b). Putting principles intro practice: understanding history. En M. Donovan, J. Bransford (eds.), How students learn: History in the classroom, 31-77, Whasington: National Academies Press.

LÉVESQUE, S. (2008). Thinking Historically. Educating Students for the 21th Century. Toronto: University of Toronto Press.

LÓPEZ FACAL, R. (2014). La LOMCE y la competencia histórica. Ayer, 94(2), 273285.

LÓPEZ FACAL, R. et al. (2011) (eds.) Pensar históricamente en tiempos de globalización. Santiago de Compostela: USC.

MACHI, C. (2014) Argumentar el presente desde la explicación histórica del pasado. Trabajo Fin de Máster de investigación en Didáctica de las Ciencias Sociales, inédito. Valencia: Universitat de València.

MERCHÁN, F. J. (2005). Enseñanza, examen y control: profesores y alumnos en la clase de historia. Barcelona: Octaedro.

MERCHÁN, F. J. (2011). Los límites y las posibilidades de la evaluación como estrategia de mejora de la práctica de la enseñanza. En P. Miralles, S. Molina, y A. Santisteban (Eds.), La evaluación en el proceso de enseñanza y aprendizaje de las Ciencias Sociales, Vol. 1, (pp. 75-84). Murcia: AUPDCS.

MERCHÁN, F. J. (2012). La introducción en España de la política educativa basada en la gestión empresarial de la escuela: el caso de Andalucía. Archivos analíticos de políticas educativas, 20(32), 1-24.

MUÑOZ-REPISO, M. (1997). El sistema de acceso a la universidad en España :tres estudios para aclarar el debate. Madrid: Ministerio de Educación y Cultura, 
Centro de Investigación y Documentación Educativa.

PAGÈS, J. (2009). El desarrollo del pensamiento histórico como requisito para la formación democrática de la ciudadanía, Reseñas de Enseñanza de la Historia, 7, 69-91.

PÉREZ GÓMEZ, A. (2013). Reválidas, evaluación de competencias y calidad de los aprendizajes. Qurriculum: Revista de teoría, investigación y práctica educativa, 26, 11-25.

PLÀ, S. (2005). Aprender a pensar históricamente. La escritura de la historia en el bachillerato. Méjico: Plaza y Valdés.

POPHAM, J. (1999). ¿Por qué las pruebas estandarizadas no miden la calidad educativa? Educational Leadership, 56(6), 2-11.

PRATS, J y SANTACANA, J. (2011). ¿Por qué y para qué enseñar historia? En Prats, J. (coord.). Didáctica de la Geografía y la Historia, 13-29. Barcelona: Graó.

RÜSEN, J. (2005). History: Narration, Interpretation, Orientation. Nueva York: Berghahn.

SÁIZ, J. (2013). Alfabetización histórica y competencias básicas en libros de texto de historia y en aprendizajes de estudiantes. Didáctica de las Ciencias Experimentales y Sociales, 27, 43-66.

SÁIZ, J. y FUSTER, C. (2014) Memorizar historia sin aprender pensamiento histórico: las PAU de Historia de España. Investigación en la escuela, 84, 47-57

SALINAS, D. (2002). ¡Mañana examen! La evaluación: entre la teoría y la realidad. Barcelona: Graó

SANMARTÍ, N. (2003). Evaluación externa: ¿por qué y para qué? Alambique: Didáctica de las ciencias experimentales(37), 9-18.

SANTISTEBAN, A. (2010). La formación de competencias de pensamiento histórico. Clío \& Asociados (14), 34-56.

SCHMIDT, M. A. y BARCA, I. (org.) (2009). Aprender história: perspectivas da educação histórica. Ijuí: ed. Unijui

SEIXAS, P. y MORTON, T. (2013). The big six historical thinking concepts. Nelson: Toronto. 
SHELDON, K.M. y BIDDLE, B.J. (1998). Standards, accountability, and school reform: perils and pifalls. Teachers College Record, 100, 1, 164-180.

SOLOMON, P.G. (2003). The assessment bridge: positive ways to link test to learning, standards, ans curriculum improvement. Corwin Press, Thousand Oaks.

SOUTO, X. M. (2011). Las PAU de Geografía: ¿¿un obstáculo o una oportunidad? En P. Miralles, S. Molina, y A. Santisteban (Eds.), La evaluación en el proceso de enseñanza y aprendizaje de las ciencias sociales. Vol.II, (pp. 271-284). Murcia: APUDCS

SOUTO, X. M. y. CLAUDINO S. (2001). Obstáculos en la innovación de la didáctica de geografía. En M. J. Marrón Gaite (Ed.), La formación geográfica de los ciudadanos en el cambio del milenio (pp. 191-203). Madrid: Asociación de Geógrafos Españoles.

SOUTO, X. M., FUSTER, C., y SÁIZ, J. (2014). Un camino de ida y vuelta: reválidas y selectividad en las rutinas escolares de la enseñanza de la Geografía e Historia. En J. Pagès y A. Santisteban (Eds.), Una mirada al pasado y un proyecto de futuro. Investigación e innovación en didáctica de las ciencias sociales Vol. 2, (pp. 157-166). Barcelona: AUPDCS.

SOUTO, X. M., FITA, S. y FUSTER, C. (2013) El control del conocimiento de Historia: exámenes y selectividad. En N. Martínez, (Dir.) La construcción de los recuerdos escolares de Historia de España en Bachillerato (1993-2013). Análisis, interpretación y poder de cambio de los testimonios de profesores y alumnos. Valencia: Nau Llibres.

TAYLOR, A. y YOUNG, C. (2003). Making History. AGuide for the Teaching and Learning of History in Australian Schools. Melbourne: Curriculum Corporation.

TRIBÓ, G. (2005). Enseñar a pensar históricamente: los archivos y las fuentes documentales en la enseñanza de la historia. Barcelona: ICE. Horsori.

VANSLEDRIGHT, B. A. (2011). The Challenge of Rethinking History Education. On Practice, Theories, and Policy. New York: Routledge.

VANSLEDRIGHT, B. A. (2014). Assessing Historical Thinking and Understanding. Innovation Design for New Standards. New York: Routledge. 
LOS ESTÁNDARES DE APRENDIZAJE DE LA LOMCE: ¿MEJORARÁN LA ENSEÑANZA Y EL APRENDIZAJE DE HISTORIA?

Carlos Fuster García

Revista de Didácticas Específicas, nº 12, PP. 27-48

WINEBURG, S. (2001). Historical Thinking and Other Unnaturals Acts: Charting the Future of Teaching the Past. Philadelphia: Temple University Press. 